\title{
Graphene-based integrated electronic, photonic and spintronic circuit
}

\author{
P. Potasz ${ }^{\text {a,c }}$, A. D. Güçlü ${ }^{\mathrm{a}, \mathrm{d}}$, I.Ozfidan ${ }^{\mathrm{a}, \mathrm{b}}$, M.Korkusinski ${ }^{\mathrm{a}}$, and P. Hawrylak ${ }^{\mathrm{a}, \mathrm{b}}{ }^{\text {}}$ \\ ${ }^{a}$ Emerging Technologies Division, National Research Council of Canada, Ottawa, K1A OR6 \\ ${ }^{\mathrm{b}}$ Department of Physics, University of Ottawa, Ottawa, Canada \\ 'Institute of Physics, Wroclaw University of Technology, Wroclaw, Poland \\ ${ }^{\mathrm{d}}$ Department of Physics, Izmir Institute of Technology, 35430 Izmir, Turkey
}

\begin{abstract}
To create carbon-based nanoscale integrated electronic, photonic, and spintronic circuit one must demonstrate the three functionalities in a single material, graphene quantum dots (GQDs), by engineering lateral size, shape, edges, number of layers and carrier density. We show theoretically that spatial confinement in GQDs opens an energy gap tunable from UV to THz, making GQDs equivalent to semiconductor nanoparticles. When connected to leads, GQDs act as singleelectron transistors. The energy gap and absorption spectrum can be tuned from UV to THz by size and edge engineering and by external electric and magnetic fields. The sublattice engineering in, e.g., triangular graphene quantum dots (TGQDs) with zigzag edges generates a finite magnetic moment. The magnetic moment can be controlled by charging, electrical field, and photons. Addition of a single electron to the charge-neutral system destroys the ferromagnetic order, which can be restored by absorption of a photon. This allows for an efficient spin-photon conversion. These results show that graphene quantum dots have potential to fulfill the three functionalities: electronic, photonic, and spintronic, realized with different materials in current integrated circuits, as well as offer new functionalities unique to graphene.
\end{abstract}

Keywords: graphene nanostructures, optical properties, magnetic properties, electronic nanodevices

\section{INTRODUCTION}

Current information technology uses different materials for storage, retrieving, transmitting, and manipulating information. This is inefficient and leads to a significant power dissipation. One approach, spintronics, attempts to merge semiconductor and ferromagnetic materials, potentially allowing logic and storage operations in the same hybrid material [1-3]. Spin injection and doping with magnetic ions of inorganic and organic semiconductors, organic ferromagnets, and carbon nanotubes were studied [4-6]. Recent progress in graphene opens new possibilities for creating multi-functional graphene-based spintronics components [7-25]. In this work, we describe electronic, photonic and magnetic properties of semiconductor nanostructures made of graphene, and discuss their potential application as building blocks for nanoscale circuits made of carbon only [26-47]. We show that fabricating graphene quantum dots with appropriate size, shape, edge termination and sublattice symmetry enables the design of semiconductor systems with magnetic and optical properties. We focus on triangular graphene quantum dots and Möbius strips with zigzag edges which combine the properties of semiconductors and ferromagnets. We also discuss the interplay between magnetic and optical properties and how they can be controlled using electric fields and photons.

\section{METHODOLOGY}

Electronic properties of graphene nanostructures within the single-particle approximation can be described using the tight-binding Hamiltonian introduced by Wallace [7]: $H=-\sum t_{i j} c_{i \sigma}{ }^{+} c_{j \sigma}$, where $c_{i \sigma}^{+}$and $c_{i \sigma}$ are creation and annihilation operators of $\mathrm{p}_{\mathrm{z}}$ electron on $i$-th site with spin $\sigma, t_{i j}$ is a hopping integral: $t=2.5 \mathrm{eV}$ between nearest and $t=0.1 \mathrm{eV}$ between next-nearest neighbors according to values for bulk graphene [12]. The tight-binding Hamiltonian describes finite size systems by applying boundary conditions at the edges, e. g., by putting a hopping integral $t=0$ to auxiliary atoms outside of the quantum dot. Such boundary conditions correspond to the hydrogen passivation of edge

*pawel.potasz@pwr.wroc.pl; pawel.hawrylak@nrc-cnrc.gc.ca

Micro- and Nanotechnology Sensors, Systems, and Applications V, edited by Thomas George,

M. Saif Islam, Achyut K. Dutta, Proc. of SPIE Vol. 8725, 87250G · C 2013 SPIE

CCC code: $0277-786 \mathrm{X} / 13 / \$ 18 \cdot$ doi: $10.1117 / 12.2016607$

Proc. of SPIE Vol. $872587250 \mathrm{G}-1$ 
carbon atoms, a situation confirmed experimentally [48]. The effect of the external magnetic field is incorporated by making the Peierls substitution [49, 50]: $t_{i j} \rightarrow t_{i j} e^{\varphi_{i j}}$, where $\varphi_{i j}$ is the magnetic field dependent phase accumulated by going from $i$-th to $j$-th site. The dimension of the Hilbert space is equal to the number of atoms because there is one electron per $p_{z}$ orbital per carbon atom. The energy eigenvalues and corresponding eigenfunctions are obtained by diagonalizing the Hamiltonian matrix numerically. Optical properties are determined by calculating dipole moments $|\langle i|\bar{r}| f\rangle|$, where $i$ is the initial and $f$ are final excited electronic states. In order to include many-electron effects in graphene quantum dots, a combination of tight-binding, Hartree-Fock and configuration interaction methods (tb-HF-CI) is used [35, 37, 38, 40, 41], with direct and exchange Coulomb matrix elements for electrons scattering up to next nearest neighbors computed using Slater $p_{z}$ orbitals. Approximate matrix elements are computed for long-range direct Coulomb interaction. Final-state interactions are included using the approximate GW-BSE approach.

\section{ELECTRONIC AND OPTICAL PROPERTIES}

Graphene is a semi-metal with a vanishing density of states at the Fermi level [12]. Opening of an energy gap in this material can be accomplished by quantum confinement, of which one possible realization is cutting graphene into quantum dots $[29,38]$. The energy spectra and opening of gaps for graphene quantum dots with different shapes and types of edges, (a) hexagonal dot with armchair edges, (b) hexagonal dot with zigzag edges, and (c) triangular dot with zigzag edges, are shown on the left-hand side of Fig. 1.
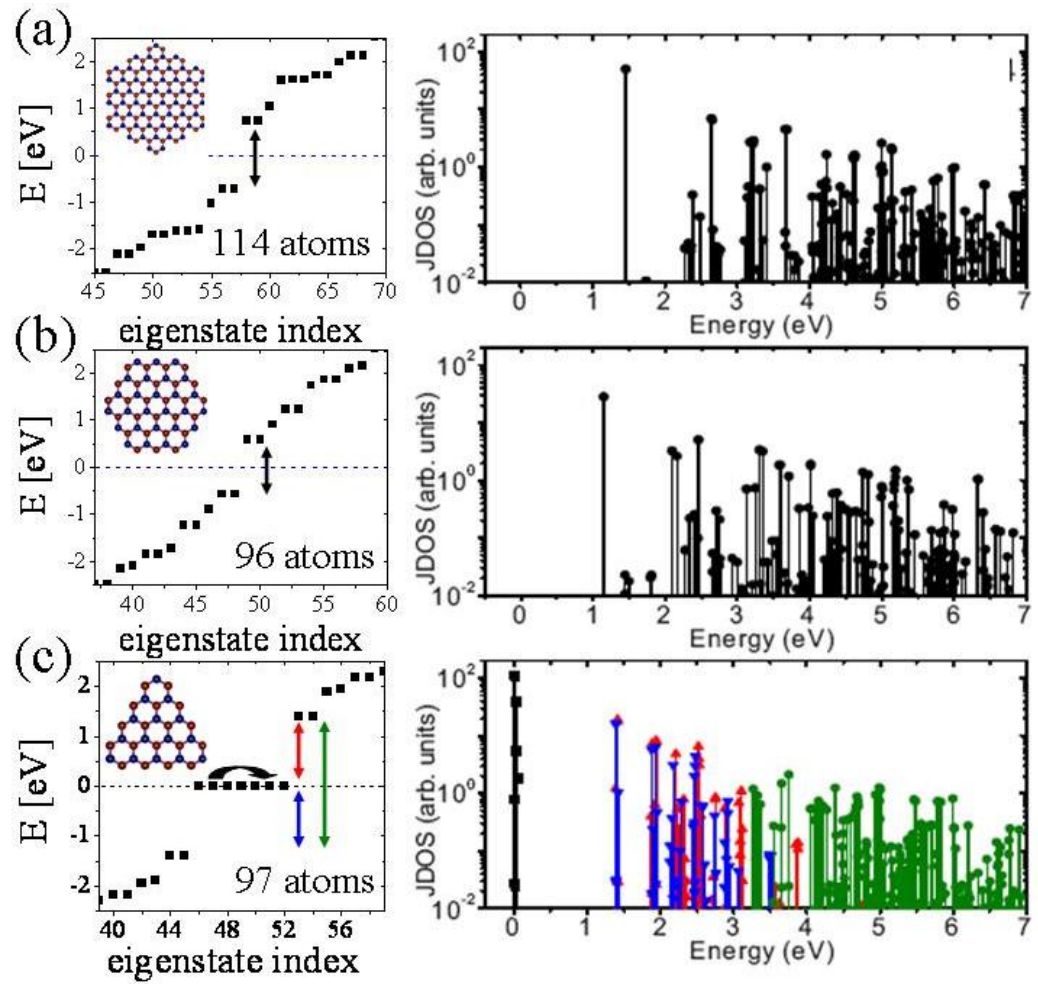

Figure 1. Left: the low-energy spectra of graphene quantum dots with different shape and edge termination consisting of N 100 atoms. The Fermi level is indicated by the blue dotted line. The energy gap is similar for all structures. In a triangular graphene quantum dot with zigzag edges, the degenerate shell of zero-energy states appears in the middle of the gap. Right: Joint optical density of states. In (a) and (b), there are transitions from the valence to conduction band, in (c) there are transitions from the valence band to the degenerate shell (blue arrow), from the degenerate shell to the conduction band (red arrow), from the valence band to the conduction band (green arrow), and within the degenerate shell (black arrow). 
The three structures have $\mathrm{N} 100$ atoms and comparable energy gaps, $\mathrm{E}_{\text {gap }} \sim 1.2 \mathrm{eV}$, indicated by black arrows in Fig. 1(a) and (b), and the green arrow in Fig. 1(c). We see that for the hexagonal structure with armchair and zigzag edges the energy spectra are similar, with the gap smaller for zigzag GQD. On the other hand, the comparison of two structures with the zigzag edge, the hexagon and the triangle, reveals a very different energy spectrum. For a TGQD there exists a shell of degenerate levels at the Fermi level. The degeneracy of this shell is a linear function of the size of the triangle and can be made macroscopic. The edge atoms belong to only one sublattice. The existence of such a shell is related to a broken sublattice symmetry in the triangle and has a profound effect on the electronic and magnetic properties to be discussed in the next Section.

Fig. 1 demonstrated the opening of a gap in the energy spectrum, with occupied valence band below the Fermi level $E_{f}$ and empty conduction band above the $\mathrm{E}_{\mathrm{f}}$. The right-hand side of Fig. 1 shows the absorption spectrum corresponding to transitions from the valence to the conduction band, showing that GQDs are optically active. The dipole moments correspond to transitions from the valence band to the conduction band for all structures, and for a triangular dot also from the valence band to the degenerate shell, from the degenerate shell to the conduction band, and between states within the degenerate shell. All structures have the largest absorption peak slightly above the energy gap. For TGQD, an extra peak at low energy appears due to transitions within the degenerate shell. Thus, graphene quantum dots, regardless of their shape and type of their edges, are optically active.

An important feature of GQDs is the ability to control the energy gap over a large energy range by controlling the size of the GQD. Fig. 2 shows the energy gap dependence on the size of the three structures from Fig.1. The energy gap is defined as the energy difference between the lowest state from the conduction band and the highest state from the valence band. Fig. 2 shows that the magnitude of the energy gap in graphene nanostructures can be designed by manipulating the size, shape and edge termination, or introducing an external magnetic field. For the hexagonal structure with zigzag edges, the energy gap rapidly vanishes when the size increases. This is related to the presence of edge states in the vicinity of the Fermi energy [29]. For TGQD and the hexagonal quantum dot with armchair edges, the energy gap is inversely proportional to the square root of the number of atoms. Such behavior is expected for Dirac Fermions with linear energy dependence on the wavevector. The energy gap varies from a few $\mathrm{eV}$ for structures consisting of tens of atoms, to a few meVs for a one million atom structure, and correspondingly from UV to THz [38]. Once the GQD is

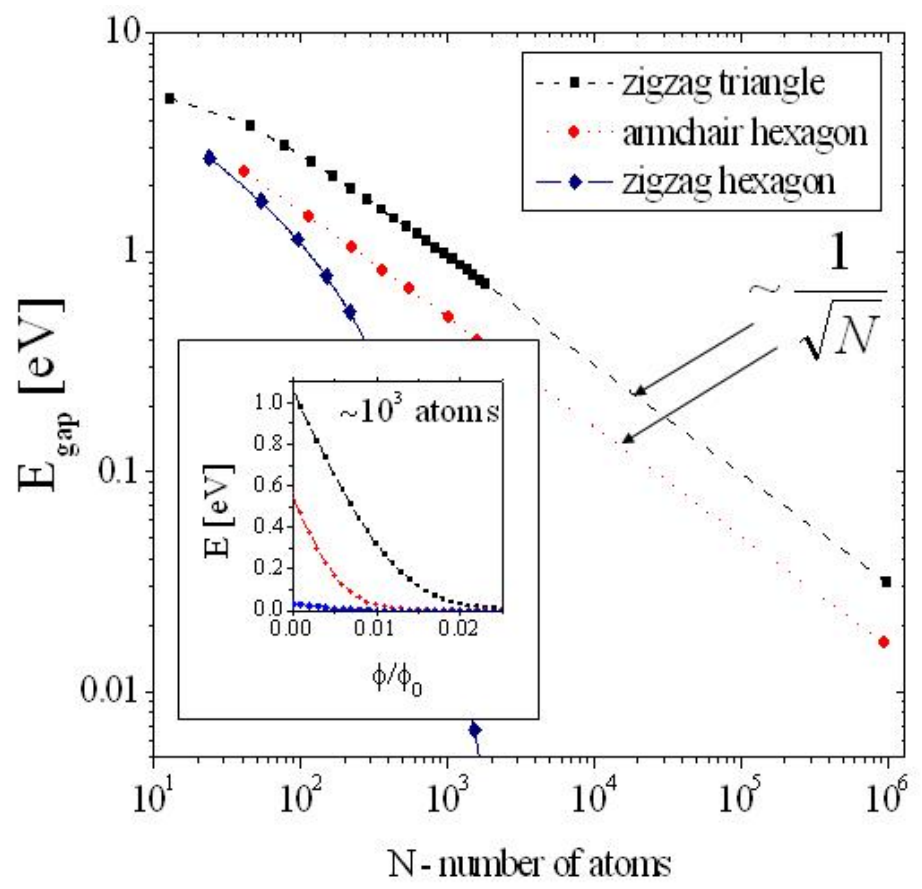

Figure 2. The energy gap as a function of size for graphene quantum dots with different shapes and edge termination, shown in Fig. 1. The inset shows closing of the energy gap when the magnetic field increases for structures consisting of around $N \sim 1000$ atoms. 
fabricated, the gap can be closed with a magnetic field. For semiconductor quantum dots the energy gap increases with the increase of the magnetic field. Inset in Fig. 2 shows the opposite behavior in GQDs: when the magnetic field increases, the energy gap closes. This is related to the existence of the zero Landau Level (0LL) exactly at the Fermi level, $\mathrm{E}=0$. In graphene nanostructures, states close to the energy gap from the valence and conduction bands evolve to OLL when the magnetic field increases, which effectively closes the energy gap. Thus, optical properties of graphene nanostructures can be tuned in a variety of ways: by changing size, shape, and edge termination, or using an external magnetic field.

\section{MAGNETIC PROPERTIES}

In order to study the magnetic properties, electron-electron interactions have to be included. They are important in particular in a system with the degenerate energy levels, like TGQD. We illustrate the magnetic properties of TGQDs on the example of a TGQD with $\mathrm{N}=97$ atoms [31-39]. There are $\mathrm{N}_{\mathrm{deg}}=7$ zero-energy states. These levels are occupied by electrons, and for the charge-neutral system the shell is half filled. Applying a metallic gate enables control of the population of electrons occupying the degenerate shell. We analyze the total spin $S$ as a function of the number of electrons in the shell, shown in Fig. 3(a). When electrons are added to the zero-energy shell one by one, the total spin increases linearly as if following the Hund's rule. For the half-filled shell, the total spin is maximal, as indicated by the red arrow in Fig. 3(a). The spin polarization for the half-filling was shown by various techniques: the Heisenberg model [31], the Hubbard model, and the density functional theory [32, 33]. Electrons within the shell are ferromagnetically coupled due to the exchange interaction. Next, one extra electron is added to the half-filled spin polarized shell. Initially it has to have spin up because all spin-down energy levels are filled. However, it turns out that this extra electron leads to a strongly correlated ground state with total spin $S=0$. The total spin $S=7 / 2$ for $N_{\mathrm{el}}=7$ electrons is decreased to $S=0$ for $\mathrm{N}_{\mathrm{el}}=8$ electrons, as shown by the blue arrow in Fig. 3(a) [35]. Removing the magnetic moment by charging with a single electron is potentially an efficient way to control magnetic properties with a gate. The robustness of the two effects observed for TGQD: (i) maximal spin polarization for the half-filled shell and (ii) the spin depolarization effect after adding one extra electron, can be confirmed by studying different systems with similar edge structure.
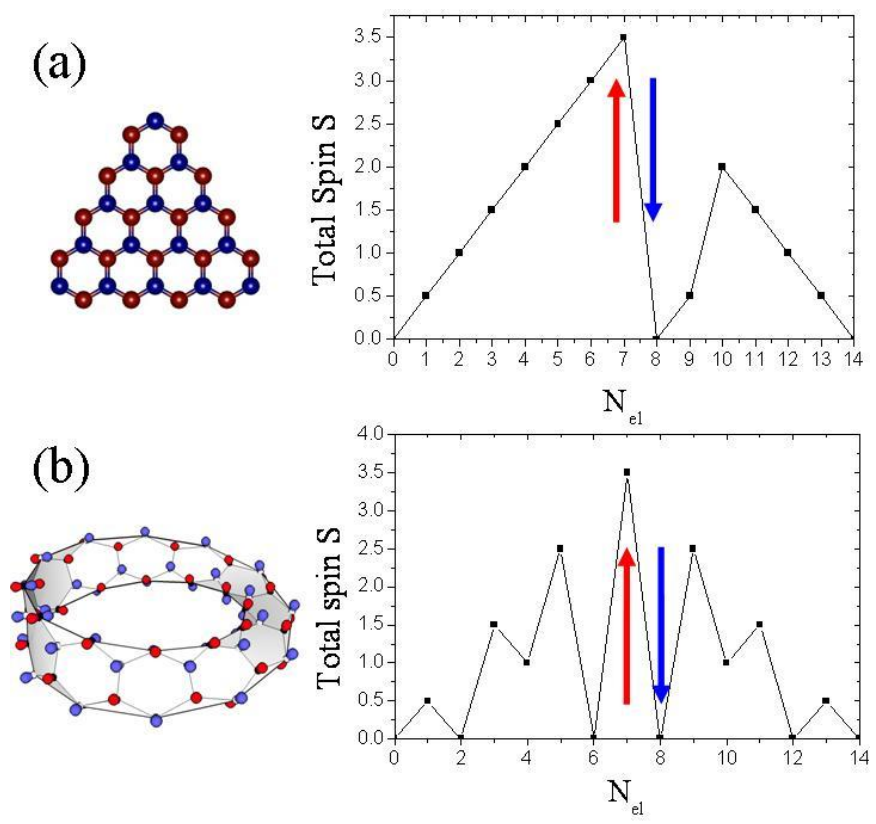

Figure 3. The total spin S as a function of the number of electrons occupying the degenerate shell for (a) triangular graphene quantum dot with zigzag edges and (b) graphene Möbius strip. For both systems, the half filled shell is maximally spin polarized (red arrow), and addition of one extra electron leads to the spin depolarization (blue arrow). 
The graphene Möbius strip shown in Fig. 3(b) on the left, due to its topology has only one edge [51] and is a prototype of a topological insulator (TI) in real space. As a TI, it has degenerate energy levels in the middle of the energy gap, with the number of states determined by the length of the strip [52]. However, in the Möbius strip the degenerate shell has a more complex structure. For the system considered here, with $\mathrm{N}=364$ atoms, there are two subshells, one with $\mathrm{N}_{\mathrm{deg}}=7$ zero-energy states, and the second nearby subshell with two extra states [52]. For the charge neutral system, there are $\mathrm{N}_{\mathrm{el}}=8$ electrons distributed among these states. When the lower subshell is half-filled with $\mathrm{N}_{\mathrm{el}}=7$ electrons, the system is maximally spin polarized. This can be understood by noting that the Möbius strip has one edge like a single edge of a graphene nanoribbon [43], or the one-sublattice edge of TGQD considered in this work. Addition of one extra electron leads to the spin depolarization, identically to the situation observed TGQD, see Fig. 4(b). Thus, effects presented here seem to be universal for electrons occupying the one-sublattice edge.

\section{COMBINING OPTICAL AND MAGNETIC PROPERTIES}

Electronic properties of triangular graphene quantum dots with zigzag edges, described in previous sections, show that these systems can be used to design an efficient semiconductor ferromagnetic device with the magnetic moment controlled by an electric gate [53]. We have shown that for the charge-neutral system, the degenerate shell of zeroenergy states is half-filled. The electron spins are aligned ferromagnetically in order to maximize the exchange interaction. This is shown in Fig. 4(a) using black downward arrows representing spin densities of $\mathrm{N}_{\mathrm{el}}=7$ electrons occupying the zero-energy edge states of the structure with $\mathrm{N}=97$ atoms. The ground state has total spin $\mathrm{S}=7 / 2$ and is separated by an energy gap from the excited states with different spins. Next an extra electron is added using an electric
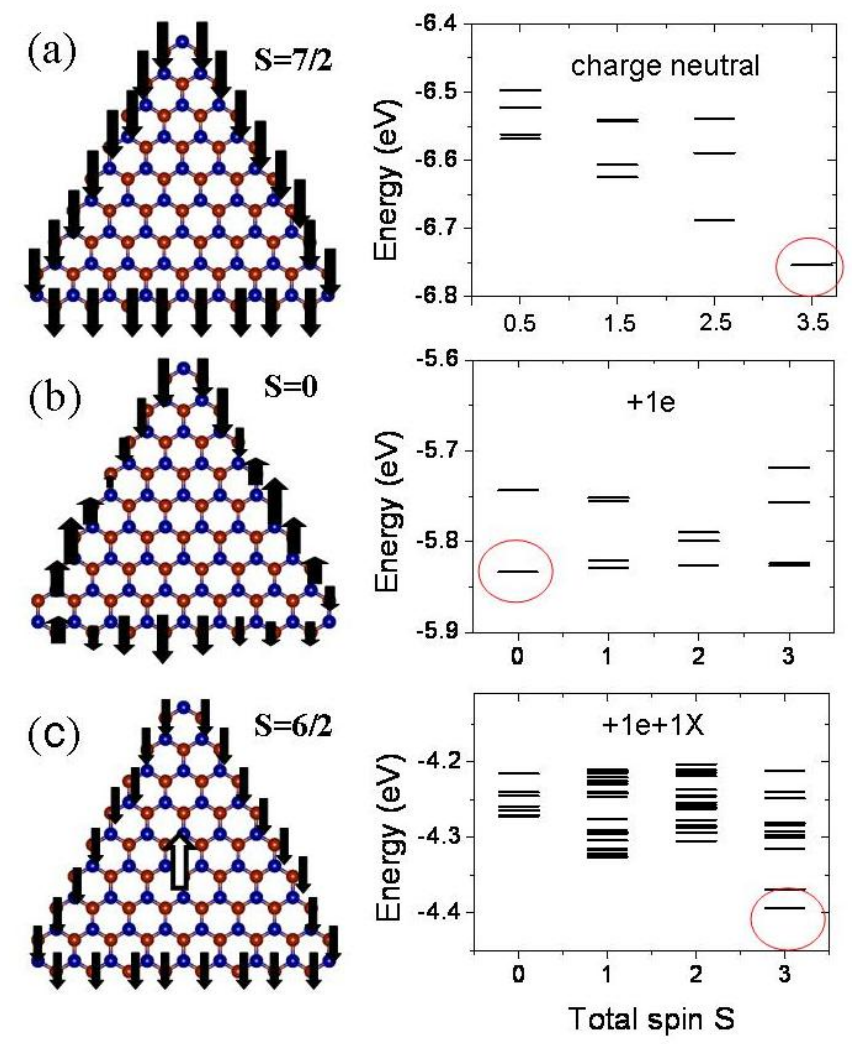

Figure 4. An efficient way of controlling the magnetic moment using an electric gate and light. On the left, black arrows represent spin densities of electrons on zero-energy edge states. On the right, many-body energy spectra for different total spins are shown, with red circles indicating the ground state. (a) The system is charge neutral with the total spin of the ground state $S=7 / 2$. (b) One extra electron is added to the system using an electric gate. The total spin of the ground state is $\mathrm{S}=0$. (c) Charged system with one extra electron and one photon absorbed. The white arrow in the center of the structure represents a hole in the valence band. The magnetic moment is restored, total spin of the ground state is $\mathrm{S}=3$. 
gate. The erromagnetic order is totally destroyed, as shown in Fig. 4(b). The total spin of the ground state is $\mathrm{S}=0$. Next, one photon is absorbed [53]. This creates an exciton with an extra electron within the degenerate shell and a hole in the valence band, indicated by the white arrow in the center of the structure shown in Fig. 4(c). The total spin of the ground state is $S=3$, thus a magnetic moment is recovered. This is related to the exchange interaction between electrons from the degenerate shell and the hole from the valence band, which overcomes correlation effects leading to the spin depolarization. Thus, the magnetization of the system is controlled by a single electron from a nearby gate and a single photon. These effects could be used to engineer the coherent spin-photon conversion process.

\section{CONCLUSIONS}

We have shown here that, at least theoretically, one can create the three functionalities: electronic, photonic, and spintronic, in a single material, graphene, by engineering the lateral size, shape, edges, and carrier density of graphene quantum dots (GQD). The future work will aim at designing ways of integrating these functionalities on a single nanoscale chip.

\section{ACKNOWLEDGEMENTS}

We would like to thank NSERC, CIFAR, and TüBİTAK for support. PP acknowledges support from the "Mistrz" program of the Foundation for Polish Science.

\section{REFERENCES}

[1] Žutić, I., Fabian, J., Sarma, S. D. "Spintronics: Fundamentals and applications", Rev. Mod. Phys., 76 (2), pp. 323-410 (2004).

[2] Dietl T., "A ten-year perspective on dilute magnetic semiconductors and oxides", Nat. Mater. 9, 965 (2010).

[3] Ohno M., Chiba D., Matsukura F., Omiya T., Abe E., Dietl T., Ohno Y., and Ohtani T., "Electric-field control of ferromagnetism", Nature (London) 408, 944 (2000).

[4] Ohno, H., "Making nonmagnetic semiconductors ferromagnetic", Science 281, 951-956 (1998).

[5] Dediu, V., Murgia M., Matacotta F. C., Taliani C., and Barbanera S., "Room temperature spin polarized injection in organic semiconductor", Solid State Commun. 122, 181-184 (2002).

[6] Tsukagoshi, K., Alphenaar B. W., and Ago H., "Coherent transport of electron spin in a ferromagnetically contacted carbon nanotube", Nature (London) 401, 572-574 (1999).

[7] Wallace P. R., "The band theory of graphite", Phys.Rev. 71, 622 (1947).

[8] Novoselov K. S., Geim A. K., Morozov S. V., Jiang D., Zhang Y., Dubonos S. V., Grigorieva I.V., and Firsov A. A., "Electric Field Effect in Atomically Thin Carbon Films", Science 306, 666 (2004).

[9] Novoselov K. S., Geim A. K., Morozov S. V., Jiang D., Katsnelson M. I., Grigorieva I. V., Dubonos S. V., and Firsov A. A., "Two-dimensional gas of massless Dirac fermions in graphene", Nature 438,197 (2005).

[10]Zhang Y. B., Tan Y.W., Stormer H.L., Kim P., "Experimental observation of the quantum Hall effect and Berry's phase in graphene", Nature 438, 201-204 (2005).

[11]Zhou S. Y., Gweon G.-H., Graf J., Fedorov A. V., Spataru C. D., Diehl R. D., Kopelevich Y., Lee D.-H., Louie S. G., and Lanzara A., "First direct observation of Dirac fermions in graphite", Nature Physics 2, 595 (2006).

[12] Castro Neto A. H., Guinea F., Peres N. M. R., Novoselov K.S., Geim A. K., "The electronic properties of graphene", Rev. Mod. Phys 81,109 (2009).

[13] Sadowski M. L., Martinez G., Potemski M., Berger C., and de Heer W. A., "Landau level spectroscopy of Ultrathin Graphite layers", Phys. Rev. Lett. 97, 266405 (2006).

[14] Nair R. R., Blake P., Grigorenko A. N., Novoselov K. S., Booth T. J., Stauber T., Peres N. M. R., Geim A. K., "Fine structure constant defines visual transparency of graphene", Science 320, 1308 (2008).

[15] Wang F., Zhang Y., Tian C., Girit C., Zettl A., Crommie M., and Shen Y. R., "Gate-variable optical transitions in graphene", Science 320, 206 (2008).

[16] Li Z. Q., Henriksen E. A., Jiang Z., Hao Z., Martin M. C., Kim P., Stormer H. L., and Basov D. N., "Dirac charge dynamics in graphene by infrared spectroscopy", Nature Physics, 532 (2008). 
[17] Mak K. F., Sfeir M. Y., Wu Y., Lui C. H., Misewich J. A., and Heinz T. F., "Measurement of the optical conductivity of graphene", Phys. Rev. Lett. 101, 196405 (2008).

[18] Xia F., Mueller T., Lin Y.-M., Valdes-Garcia A., and Avouris P., "Ultrafast graphene photodetector", Nature Nanotechnology 4,839 (2009).

[19] Mueller T., Xia F., and Avouris P., "Graphene photodetectors for high-speed optical communications", Nat. Photonics, 4, 297 (2010).

[20]Ritter K. A. and Lyding J. W., "The influence of edge structure on the electronic properties of graphene quantum dots and nanoribbons", Nature Mater. 8, 235 (2009).

[21] Yang L., Deslippe J., Park C.-H., Cohen M. L., and Louie S. G., "Excitonic effects on the optical response of graphene and bilayer graphene", Phys. Rev. Lett. 103, 186802 (2009).

[22] Bunch J. S., Yaish Y., Brink M., Bolotin K., and McEuen P. L., "Coulomb Oscillations and Hall Effect in Quasi-2D Graphite Quantum Dots", Nano Letters 5, 287 (2005).

[23] Ihn T., Gustavsson S., Gasser U., Küng B., Müller T., Schleser R., Sigrist M., Shorubalko I., Leturcq R., and Ensslin K., "Quantum dots investigated with charge detection techniques", Solid St. Comm. 149, 1419 (2009).

[24] Ponomarenko L. A., Schedin F., Katsnelson M. I., Yang R., Hill E. W., Novoselov K. S., Geim A. K., "Chaotic Dirac Billiard in Graphene Quantum Dots", Science 320, 356 (2008).

[25] Campos L. C., Manfrinato V. R., Sanchez-Yamagishi J. D., Kong J., and Jarillo-Herrero P., "Anisotropic Etching and Nanoribbon Formation in Single-Layer Graphene", Nano Lett., 9, 2600 (2009).

[26] Wunsch B., Stauber T., Guinea F., "Electron-electron interactions and charging effects in graphene quantum dots", Phys Rev B 77, 035316 (2008).

[27]Wurm J. J., Rycerz A., Adagideli I., Wimmer M., Richter K., and Baranger H. U., "Symmetry Classes in Graphene Quantum Dots: Universal Spectral Statistics, Weak Localization, and Conductance Fluctuations", Phys. Rev. Lett. 102, 056806 (2009).

[28] Libisch F., Stampfer C., and Burgdorfer J., "Graphene quantum dots: Beyond a Dirac billiard", Phys. Rev. B 79, 115423 (2009).

[29]Zhang Z. Z., Chang Kai, and Peeters F. M., "Tuning of energy levels and optical properties of graphene quantum dots", Phys. Rev. B 77, 235411 (2008).

[30] Mueller M. L., Yan X., McGuire J. A., and Li L.-S., "Triplet States and Electronic Relaxation in Photoexcited Graphene Quantum Dots", Nano Lett. 10, 2679 (2010).

[31] Ezawa M., "Coulomb blockade in graphene nanodisks", Phys. Rev. B 77, 155411 (2008); Ezawa M., "Metallic graphene nanodisks: Electronic and magnetic properties", Phys. Rev. B 76, 245415 (2007).

[32] Fernandez-Rossier J., and Palacios J. J., "Magnetism in graphene nanoislands", Phys. Rev. Lett. 99, 177204 (2007).

[33] Wang W. L., Meng S., Kaxiras E., "Graphene nanoflakes with large spin", Nano Lett. 8, 241 (2008).

[34] Akola J., Heiskanen H. P., and Manninen M., "Edge-dependent selection rules in magic triangular graphene flakes", Phys. Rev. B 77, 193410 (2008).

[35] Güçlü A. D., Potasz P., Voznyy O., Korkusinski M., and Hawrylak P., "Magnetism and correlations in fractionally filled degenerate shells of graphene quantum dots", Phys Rev. Lett. 103, 246805 (2009).

[36] Potasz P., Güçlü A. D., Hawrylak P., "Zero-energy states in triangular and trapezoidal graphene structures", Phys.Rev.B 81, 033403 (2010).

[37] Potasz P., Güçlü A. D., Hawrylak P., "Electronic properties of gated triangular graphene quantum dots: Magnetism, correlations, and geometrical effects", Phys. Rev. B, 85, 075431 (2012).

[38] Güçlü A. D., Potasz P., and Hawrylak P., "Excitonic absorption in gate-controlled graphene quantum dots", Phys. Rev. B, 82, 155445 (2010).

[39] Voznyy O., Güçlü A. D., Potasz P., Hawrylak P., "Effect of edge reconstruction and passivation on zero-energy states and magnetism in triangular graphene quantum dots with zigzag edges" Phys. Rev. B, 83, 165417 (2011).

[40] Potasz P., Güçlü A. D., Voznyy O., Folk J., and Hawrylak P., "Electronic and magnetic properties of triangular graphene quantum rings", Phys. Rev. B, 83, 174441 (2011).

[41] Güçlü A. D., Potasz P., and Hawrylak P., "Electric-field controlled spin in bilayer triangular graphene quantum dots", Phys. Rev. B, 84, 035425 (2011).

[42] Nakada K., Fujita M., Dresselhaus G., and Dresselhaus M. S., "Edge state in graphene ribbons: Nanometer size effect and edge shape dependence", Phys. Rev. B 54, 17954 (1996).

[43] Yamashiro A., Shimoi Y., Harigaya K., and Wakabayashi K., "Spin- and charge-polarized states in nanographene ribbons with zigzag edges", Phys. Rev. B 68, 193410 (2003). 
[44] Yang L., Cohen M. L., and Louie S. G., "Magnetic Edge-State Excitons in Zigzag Graphene Nanoribbons", Phys. Rev. Lett. 101, 186401 (2008).

[45] Jung J. and MacDonald A. H., "Carrier density and magnetism in graphene zigzag nanoribbons", Phys. Rev. B 79, 235433 (2009).

[46] Brey L. and Fertig H. A., "Electronic states of graphene nanoribbons studied with the Dirac equation", Phys. Rev. B, 73, 235411 (2006).

[47] Kouwenhoven L. P., Austing D. G., and Tarucha S., "Few-electron quantum dots", Rep. Prog. Phys. $64701-$ 736 (2001).

[48] Zhang X., Yazyev O. V., Feng J., Xie L., Tao C., Chen Y., Jiao L., Pedramrazi Z., Zettl A., Louie S. G., Dai H., and Crommie M. F., "Experimentally Engineering the Edge Termination of Graphene Nanoribbons", ACS Nano 7, 198 (2013).

[49] Peierls R. E., "Zur Theorie des Diamagnetismus von Leitungselektronen", Z. Phys. 80, 763 (1933).

[50] Luttinger J. M., "The Effect of a Magnetic Field on Electrons in a Periodic Potential", Phys. Rev. 84, 814 (1951).

[51]Herges R., "Topology in chemistry: Designing Möbius molecules", Chem. Rev. 106, 4820 (2006).

[52] Güçlü A. D., Grabowski M., and Hawrylak P., "Electron-electron interactions and topology in the electronic properties of gated graphene nanoribbon rings in Möbius and cylindrical configurations", Phys. Rev. B, 87, 035435 (2013).

[53] Güçlü A. D., and Hawrylak P., "Optical control of magnetization and spin blockade in graphene quantum dots", Phys. Rev. B, 87, 035425 (2013). 